\title{
Role of regorafenib as second-line therapy and landscape of investigational treatment options in advanced hepatocellular carcinoma
}

This article was published in the following Dove Press journal:

Journal of Hepatocellular Carcinoma

21 September 2016

Number of times this article has been viewed

\author{
Jörg Trojan \\ Oliver Waidmann \\ Medizinische Klinik I, \\ Universitätsklinikum Frankfurt, \\ Germany
}

Correspondence: Jörg Trojan Medizinische Klinik I,

Universitätsklinikum Frankfurt, TheodorStern-Kai 7, 60590 Frankfurt am Main,

Germany

Tel +496963017860

Fax +4969630I 83776

Email trojan@em.uni-frankfurt.de

\begin{abstract}
Sorafenib is still the only systemic drug approved for the treatment of advanced hepatocellular carcinoma (HCC). In recent years, several investigational agents mainly targeting angiogenesis failed in late-phase clinical development due to either toxicity or lack of benefit. Recently, data of the RESORCE trial, a placebo-controlled Phase III study that evaluated the efficacy and safety of regorafenib in patients with HCC and documented disease progression after systemic first-line treatment with sorafenib, were presented at the ESMO World Congress on Gastrointestinal Cancer, 2016. Regorafenib treatment resulted in a 2.8-month survival benefit compared to placebo (10.6 months vs 7.8 months). Side effects were consistent with the known profile of regorafenib. The approval of regorafenib for this indication is expected in 2017. Further candidate agents in Phase III evaluation for second-line treatment of patients with HCC are the MET inhibitors tivantinib and cabozantinib, the vascular endothelial growth factor receptor-2 antibody ramucirumab, and the programmed death receptor-1 (PD-1) blocking antibody pembrolizumab. Furthermore, results from two first-line trials with either the tyrosine kinase inhibitor lenvatinib or the PD-1 antibody nivolumabin in comparison to sorafenib are awaited in the near future and might further change the treatment sequence of advanced HCC. Keywords: hepatocellular carcinoma, receptor tyrosine kinase inhibitor, sorafenib, regorafenib, lenvatinib, tivantinib, cabozantinib, ramucirumab, immunotherapy, anti-CTLA-4, anti-PD-1, oncolytic virus
\end{abstract}

\section{Introduction}

The multityrosine kinase inhibitor sorafenib is still the only systemic drug, which prolongs overall survival (OS) in advanced stage hepatocellular carcinoma (HCC) patients, and currently it is the standard systemic treatment option in patients with locally advanced HCC not amendable to locoregional treatment or in patients with metastatic disease. ${ }^{1,2}$ Within the last few years, several Phase III trials have investigated other tyrosine kinase inhibitors (TKIs) mainly targeting angiogenesis in comparison to sorafenib in first-line treatment. ${ }^{3}$ However, none of these drugs, such as sunitinib, brivanib, linifanib, and the combination of sorafenib and erlotinib, was superior to sorafenib in terms of OS or toxicity. ${ }^{4-7}$ Furthermore, until today there has been no established systemic second-line treatment option in patients who have progressed on sorafenib treatment. ${ }^{8}$ Currently, several agents with different targets are investigated in clinical trials in patients with advanced HCC. In this review, recent data on antiangiogenic agents including the multikinase inhibitors sorafenib and regorafenib, inhibitors of the MET pathway, and immunotherapeutics will be discussed. 


\section{Antiangiogenic agents Sorafenib}

Sorafenib is an oral multikinase and angiogenesis inhibitor with activity against vascular endothelial growth factor receptor (VEGFR)-2, platelet-derived growth factor receptor- $\beta$ (PDGFR- $\beta$ ), c-Kit receptors, BRAF, and p38 signaling pathways. It is the only approved systemic treatment option in advanced stage HCC. The high recurrence rate of HCC after resection of local ablation was the rationale for the initiation of the STORM trial, which tested adjuvant treatment with sorafenib vs placebo after R0 resection, or complete ablation of HCC. However, sorafenib did not show an improvement in progression-free survival in this Phase III trial and was associated with substantial side effects comparable to adverse events reported in advanced stage HCC patients. ${ }^{9}$ Therefore, adjuvant treatment with sorafenib should not be recommended in patients after resection or ablation.

\section{Regorafenib}

Regorafenib is a novel diphenylurea multikinase inhibitor of VEGFR1-3, c-KIT, TIE-2, PDGFR- $\beta$, FGFR-1, RET, c-RAF, BRAF, and $\mathrm{p} 38 \mathrm{MAP}$ kinase. ${ }^{10}$ Although it is structurally related to sorafenib (Figure 1), the addition of a fluorine atom in the central phenyl ring might result in a higher potency. It has been approved for the treatment of metastatic colorectal cancer after failure of oxaliplatin- and irinotecan-based systemic chemotherapy and shows a significant prolongation of OS compared to placebo. ${ }^{11}$ Moreover, it is approved for the treatment of metastatic gastrointestinal stroma tumors after failure of imatinib and sunitinib. ${ }^{12}$ A small single-arm Phase II study in HCC patients who progressed on sorafenib ( $\mathrm{n}=36$ ) was reported that showed a signal for activity. ${ }^{13}$ In this Phase II trial, the median OS was 13.8 months, and the efficacy was mainly based on disease stabilization with a disease control rate of $72 \%$. In this trial, the side effect profile of regorafenib seemed quite similar to sorafenib, such as hypertension, hand-foot skin reaction, fatigue, and diarrhea. Of note, none of the deaths including two patients who died due to liver failure were deemed to be related to regorafenib.

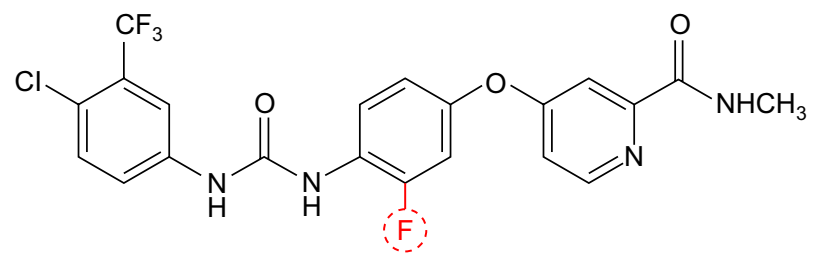

Figure I Chemical structure of sorafenib: 4-(4-[\{(4-chloro-3-[trifluoromethyl] phenyl)carbamoyl\}amino]-3-phenoxy)- $\mathrm{N}$-methylpyridine-2-carboxamide.

Note: Regorafenib differs from sorafenib by addition of a fluorine atom in the central phenyl ring as indicated in red, resulting in different target inhibition.
In early July 2016, data from a Phase III trial (RESORCE) of regorafenib in patients who progressed under sorafenib treatment (NCT01774344) have been reported as late-breaking abstract at the ESMO World Congress on Gastrointestinal Cancer in Barcelona. ${ }^{14}$ This trial enrolled patients with HCC stage BCLC B or C with preserved liver function (Child-Pugh A) and good performance status (ECOG 0 or 1). The patients had to tolerate treatment with sorafenib (400 $\mathrm{mg}$ bid) for at least 20 days and the reason for termination of sorafenib had to be documented disease progression. After a maximum of 10 weeks following the end of sorafenib treatment, patients had to be enrolled in the RESORCE trial. A total of 573 patients were randomized to receive either treatment with regorafenib (160 mg qd from day 1 to day 21 of a 28-day cycle) or placebo (2:1 randomization). The primary end point of this study was OS, which was clearly met for patients in the regorafenib arm (median OS 10.6 months vs 7.8 months, hazard ratio [HR] 0.62 ; $95 \%$ CI $0.50-0.78$; $P<0.001)$. The median progression-free survival was also superior for patients treated with regorafenib (3.1 months vs 1.5 months). Response rates (mRECIST for HCC) were also higher for regorafenib $(10.6 \%$ vs $4.1 \%){ }^{4}$

The most common grade $\geq 3$ adverse events in the regorafenib group were hypertension (15.2\%), hand-foot skin reaction $(12.6 \%)$, fatigue $(9.1 \%)$, and diarrhea (3.2\%), which is consistent with the known profile of regorafenib colorectal cancer and gastrointestinal stroma tumors. The approval of regorafenib for this indication is expected in 2017.

\section{Lenvatinib}

Lenvatinib is a multitargeted TKI of the VEGFRs 1, 2, and 3, FGFRs $1-4$, PDGFR $\alpha$, RET, and KIT signaling networks. ${ }^{15}$ In patients with differentiated thyroid cancer refractory to radioiodine (iodine-131) therapy, lenvatinib showed an impressive improvement in progression-free survival in a recent trial. ${ }^{15}$ The $\mathrm{HR}$ for progression or death was 0.21 with a 99\% CI interval of $0.14-0.31(P<0.001) .{ }^{15}$ Additionally, nearly two-thirds of the patients (64.8\%) showed an objective response to the lenvatinib treatment according to RECIST 1.1 assessment including four complete responses. The most common treatment-related adverse events were similar to other TKIs including hypertension, diarrhea, and fatigue. Due to adverse events, $14.2 \%$ of the patients had to withdraw treatment with lenvatinib in this trial. Recent data of a Phase $\mathrm{Ib}$ dose escalation trial with lenvatinib in advanced HCC showed an encouraging response rate of $15 \%$ with tumor shrinkage in 14 of 20 patients. ${ }^{16}$ Currently, a worldwide Phase III trial 
is investigating the safety and efficacy of lenvatinib in comparison to sorafenib in the first-line setting (NCT01761266). First data are awaited in late 2016.

\section{Ramucirumab}

Ramucirumab is a monoclonal antibody targeting the VEGFR 2 and was shown to improve OS as monotherapy or in combination with paclitaxel in patients receiving second-line treatment for metastatic gastric cancer. ${ }^{17,18} \mathrm{~A}$ recent Phase III study $\left(\mathrm{REACH}^{19}\right)$ comparing ramucirumab with placebo in patients after failure of sorafenib missed its primary end point. The OS in the intention-to-treat population $(n=565)$ was not significantly different between the ramucirumab and the placebo arm (HR $0.87,95 \%$ CI $0.72-1.05 ; P=0.14$; median OS 9.2 months for ramucirumab vs 7.6 months for placebo). Nevertheless, ramucirumab resulted in a robust PFS improvement compared to placebo (HR 0.63, 95\% CI 0.52 $0.75 ; P<0.001$; median PFS 2.8 months for ramucirumab vs 2.1 months for placebo) in the intention-to-treat population, without any safety concerns. However, in the subgroup of patients with baseline AFP $\geq 400 \mathrm{ng} / \mathrm{mL}$ ( $\mathrm{n}=250$ ), the OS was significantly longer for the patients treated with ramucirumab (HR $0.67,95 \%$ CI $0.51-0.90 ; P=0.0059$ ) with a median OS of 7.8 months for ramucirumab and 4.2 months for placebo. ${ }^{19}$ This confirms that high AFP is a negative prognostic factor. The reason why ramucirumab is more active in this situation is not clear yet. Thus, ramucirumab was currently tested in a new Phase III trial (NCT02435433) in HCC patients with elevated AFP after failure of sorafenib (either progression or intolerance).

\section{MET inhibitors}

MET is the receptor for hepatocyte growth factor, which is one of the predominant factors involved in liver regeneration and wound healing. ${ }^{20,21}$ Overexpression of MET is found in $25 \%-87 \%$ of $\mathrm{HCC}$ patients, and especially in patients with advanced stages and vascular invasion the prevalence of high MET expression is most frequent. ${ }^{22}$ MET overexpression was shown to be a negative prognostic factor in $\mathrm{HCC}$ patients after failure of sorafenib therapy. ${ }^{23}$ In HCC, two small molecules are currently evaluated in Phase III trials, tivantinib and cabozantinib.

\section{Tivantinib}

Tivantinib is a non-ATP competitive MET inhibitor, which selectively binds to the canonical autoinhibited conformation of MET, which is only present at the inactive, unphosphorylated form of MET. ${ }^{24,25}$ It shows only modest off-target inhibition of Flt4, CAMKIIS, PAK3, and Pim-1 kinases. Besides, there is no binding to epidermal growth factor receptor, insulin receptor, platelet-derived growth factor receptor $\alpha$, and fibroblast growth factor receptor kinases. ${ }^{25}$ In a Phase II study, tivantinib improved the progression-free survival in comparison to placebo in a Phase II randomized controlled trial (HR 0.64, 90\% CI 0.43-0.94; $P=0.04$ ). ${ }^{26}$ Patients with high MET expression had a substantial benefit from tivantinib. The median OS time was 7.2 months (95\% CI 3.9-14.6) in patients with high MET expressing tumors who received tivantinib vs 3.8 months (95\% CI 2.1-6.8) for MET-high patients who were on placebo (HR $0.38,95 \%$ CI $0.18-0.81$; $P=0.01 .^{26}$ The Phase III trial investigating tivantinib is currently recruiting for advanced HCC (NCT01755767).

\section{Cabozantinib}

Cabozantinib is a receptor TKI with activity against MET, VEGFR2, FLT3, c-KIT, and RET. ${ }^{27}$ It improves the progression-free survival in patients with refractory medullary thyroid cancer and was recently approved in this condition. ${ }^{28}$ However, recently the Phase III study of cabozantinib in pretreated metastatic castration-resistant prostate cancer patients failed to show a benefit in OS in comparison to prednisone. ${ }^{29}$ Cabozantinib was also investigated in $\mathrm{HCC}$ patients in a Phase II clinical trial. ${ }^{30}$ The overall disease control rate at 12 weeks was $68 \%$ with two partial responses. The observed effects were independent from prior sorafenib therapy. The common side effects were similar to other TKIs with diarrhea $(17 \%)$, palmar-plantar erythrodysesthesia (15\%), and thrombocytopenia $(10 \%)$ being the most common grade 3/4 adverse events. Currently, a Phase III trial investigating cabozantinib to placebo following progression or intolerance to sorafenib is recruiting HCC patients (NCT01908426).

\section{Immunotherapeutics}

Immunotherapeutics are very promising therapeutic tools in many advanced cancers. For advanced melanoma, the monoclonal human T-lymphocyte-associated antigen 4 antibody (anti-CTLA-4) ipilimumab was approved in 2011, and very recently, nivolumab and pembrolizumab, both programmed death receptor-1 (PD-1) blocking antibodies, were also approved for this disease. HCC is a very attractive candidate for immunotherapy. This is based on the report of some cases with spontaneous regression and on occasional objective tumor responses after adoptive immunotherapy with, eg, dendritic cells. ${ }^{31-33}$ Interestingly, some cases of spontaneous regression were associated with systemic inflammatory response, eg, activation of 
CD163+ macrophages was reported in vital tumor cells only but not in necrotic tumor areas. ${ }^{32}$ Currently, different classes of immunotherapeutics are in clinical development for advanced HCC.

\section{Tremelimumab}

Blockade of immune checkpoints is well established as therapeutic approach in advanced melanoma. The CTLA-4 antibody ipilimumab was approved for advanced melanoma in 2011, and treatment is associated with long-term survival in $20 \%-25 \%$ of these patients. ${ }^{34}$ Tremelimumab is another CTLA-4 antibody in clinical development, and it has been investigated in a Phase Ib trial in HCC. ${ }^{35}$ This trial enrolled 21 patients with HCC and chronic hepatitis $\mathrm{C}$ virus infection. The study included patients with ChildPugh A ( $n=12)$, as well as Child-Pugh B cirrhosis $(n=9)$. All patients included were not candidates for locoregional treatments, and concomitant antiviral treatment was not allowed. Tremelimumab was administered every 90 days at a dose of $15 \mathrm{mg} / \mathrm{kg}$ intravenously until progression or intolerable toxicity. First of all, tremelimumab was well tolerated without deterioration of liver function, although grade $3 / 4$ liver enzyme elevations were noticed in $45 \%$ of patients. The objective response rate was $17.6 \%$, and $76.4 \%$ of patients achieved disease stabilization. The time to tumor progression was 6.5 months (95\% CI 3.95-9.14), and median OS was 8.2 months (95\% CI 4.64-21.34). Moreover, treatment with tremelimumab induced a significant decrease in viral load, and three patients had a transient complete viral response during follow-up. Thus, antiCTLA-4 blockade seems to have antiviral effects in addition to antitumor efficacy, which warrant further investigation in larger clinical trials. The results of a Phase I clinical trial of tremelimumab with transarterial chemoembolization or ablation were presented at ASCO 2016 (NCT01853618) and showed encouraging results. ${ }^{36}$

\section{Nivolumab}

PD-1 and its ligands, PD-1 ligand 1 (PD-L1) and 2 (PD-L2), play important roles in the regulation of immune responses. This inhibitory action might help in immune evasion by cancer cells as PD-1, PD-L1, and PD-L2 are abnormally expressed by tumor cells and tumor-infiltrating lymphocytes. Nivolumab and pembrolizumab, both PD-1 antibodies, have been approved for advanced melanoma at the end of 2014 . Clinical trials have shown high (up to 40\%) and lasting tumor responses, even in ipilimumab pretreated advanced melanoma patients. ${ }^{37,38}$ For $\mathrm{HCC}$, no results have been reported yet. Currently, a Phase I/II trial with nivolumab in advanced HCC is recruiting patients (NCT01658878). The first part of this trial is a dose escalation phase designed to establish the safety of the drug at different dose levels for three cohorts: HCC patients without viral hepatitis, HCC patients with chronic hepatitis $\mathrm{C}$ virus infection, and $\mathrm{HCC}$ patients with chronic hepatitis B virus infection. The second part of the study is an expansion phase designed to generate additional clinical data at specified doses for each of the three cohorts plus a head-to-head comparison against sorafenib and a nivolumab/ipilimumab combination arm. Moreover, a Phase III trial recruiting patients in the first-line setting is currently enrolling patients.

\section{Oncolytic viruses}

Oncolytic viruses are a promising class of drugs that preferentially replicate in cancer cells as well as finally kill the malignant cells. There is growing evidence that viruses are unlikely to cause direct cell death, but merely favor efficient and antitumor immune response..$^{39}$ In HCC, several oncolytic viruses have been investigated in Phase I and II trials. ${ }^{40}$ The current lead agent is JX-594, which is also known as pexastimogene 60 devacirepvec (Pexa-Vec).$^{41}$ An additional oncolytic in early development in HCC is talimogene laherparepvec (T-VEC; NCT02509507).

JX-594 is a vaccinia virus (Wyeth vaccine strain) with disruption of the viral $T K$ gene for cancer selectivity and insertion of human granulocyte-macrophage colony-stimulating factor and $\beta$-galactosidase transgenes for immune stimulation and replication assessment, respectively. JX-594 is aimed to induce virus replication-dependent lysis of tumor cells as well as to induce tumor-specific immunity. In a Phase II clinical trial, JX-594 was administered in two doses: low-dose $\left(10^{8} \mathrm{PFU}\right)$ and high-dose ( $\left.10^{9} \mathrm{PFU}\right) \mathrm{JX}-594$ in patients with advanced HCC. The study was terminated early as patients receiving higher doses of JX-954 showed a significantly longer OS, namely 14.1 months for the high-dose group compared to 6.7 months in the low-dose group. Main side effects were flu-like symptoms such as pyrexia and chills, mainly grade 2, which were found in all patients. Grade 3/4 events were less frequent and manageable..$^{42}$ Currently, a Phase III trial of JX-594 in combination with sorafenib vs sorafenib as first-line treatment is enrolling patients (PHOCUS trial, NCT02562755).

\section{Conclusion}

Regorafenib is the second drug, which proved to be efficacious in patients with advanced HCC. The RESORCE trial 
clearly demonstrated that regorafenib after progression on sorafenib is active with a manageable safety profile. After several negative clinical trials in advanced HCC, this trial has shown that selection is the key. Especially, in a disease like HCC, in which prognosis is dependent on tumor biology and liver function, the strategy to include only patients who could tolerate treatment with sorafenib was the most likely reason why this trial is positive. All other clinical trials in second-line included patients irrespective of the cause of sorafenib failure (either progress or intolerance). For clinical practice, this means that after sorafenib progression treatment with regorafenib will become the standard of care. This also has implications for ongoing and future trials in the second-line setting. If the magnitude of benefit for second-line investigated drugs is in the range of regorafenib, differences in tolerability will be a major argument for or against a drug. In the next 18 months, data of several ongoing first-line and second-line trials will become available and might further change the care of patients with advanced HCC (Table 1).

\section{Disclosure}

Jörg Trojan received consulting and/or lecture fees from Amgen, Bayer Healthcare, Bristol Myers-Squibb, Daichi Sankyo, Merck Serono, Merck Sharp \& Dohme, and Lilly Imclone. Oliver Waidmann received consulting and/or lecture fees from Bayer Healthcare, Merck Serono, Novartis Oncology, and Roche. The authors report no other conflicts of interest in this work.

Table I Landscape of systemic treatment in hepatocellular carcinoma

\begin{tabular}{|c|c|c|c|c|}
\hline Agent & Class & $\begin{array}{l}\text { Line of } \\
\text { treatment }\end{array}$ & Status & Result \\
\hline Sorafenib & TKI & First line & SOC & $\begin{array}{l}\text { Median OS } \\
10.7 \text { months }\end{array}$ \\
\hline Lenvatinib & TKI & First line & Phase III & Q4 2016 \\
\hline Nivolumab & anti-PD-I & First line & Phase III & Q2 2017 \\
\hline JX-594 & $\begin{array}{l}\text { Oncolytic } \\
\text { virus }\end{array}$ & $\begin{array}{l}\text { First line in } \\
\text { combination } \\
\text { with sorafenib }\end{array}$ & Phase III & Q12018 \\
\hline Regorafenib & TKI & Second line & Phase III ${ }^{\mathrm{a}}$ & $\begin{array}{l}\text { Median OS } \\
10.6 \text { months }\end{array}$ \\
\hline Tivantinib & anti-MET & Second line & Phase III & Q4 2016 \\
\hline Cabozantinib & anti-MET & Second line & Phase III & Q2-3 2017 \\
\hline Ramucirumab & anti-VEGR2 & Second line & Phase III & Q2-3 2017 \\
\hline Pembrolizumab & anti-PD-I & Second line & Phase III & QI 2018 \\
\hline
\end{tabular}

Note: ${ }^{a}$ RESORCE trial with positive results, approval expected in 2017.

Abbreviations: OS, overall survival; TKI, tyrosine kinase inhibitor; SOC, standard of care; anti-PD-I, antiprogrammed cell death receptor I blocking antibody; anti-VEGFR2, anti-vascular endothelial growth factor receptor 2 blocking antibody.

\section{References}

1. Llovet JM, Ricci S, Mazzaferro V, et al; SHARP Investigators Study Group. Sorafenib in advanced hepatocellular carcinoma. NEngl JMed. 2008;359(4):378-390.

2. European Association For The Study OfThe Liver, European Organisation For Research And Treatment Of Cancer. EASL-EORTC clinical practice guidelines: management of hepatocellular carcinoma. J Hepatol. 2012;56(4):908-943.

3. Welker MW, Trojan J. Antiangiogenic treatment in hepatocellular carcinoma: the balance of efficacy and safety. Cancer Manag Res. 2013;5:337-347.

4. Cheng AL, Kang YK, Lin DY, et al. Sunitinib versus sorafenib in advanced hepatocellular cancer: results of a randomized phase III trial. J Clin Oncol. 2013;31(32):4067-4075.

5. Llovet JM, Decaens T, Raoul JL, et al. Brivanib in patients with advanced hepatocellular carcinoma who were intolerant to sorafenib or for whom sorafenib failed: results from the randomized phase III BRISK-PS study. J Clin Oncol. 2013;31(28):3509-3516.

6. Cainap C, Qin S, Huang WT, et al. Linifanib versus Sorafenib in patients with advanced hepatocellular carcinoma: results of a randomized phase III trial. J Clin Oncol. 2015;33(2):172-179.

7. Zhu AX, Rosmorduc O, Evans TR, et al. SEARCH: a phase III, randomized, double-blind, placebo-controlled trial of sorafenib plus erlotinib in patients with advanced hepatocellular carcinoma. J Clin Oncol. 2015; 33(6):559-566.

8. Llovet JM. Liver cancer: time to evolve trial design after everolimus failure. Nat Rev Clin Oncol. 2014;11(9):506-507.

9. Bruix J, Takayama T, Mazzaferro V, et al; STORM investigators. Adjuvant sorafenib for hepatocellular carcinoma after resection or ablation (STORM): a phase 3, randomised, double-blind, placebo-controlled trial. Lancet Oncol. 2015;16(13):1344-1354.

10. Strumberg D, Schultheis B. Regorafenib for cancer. Expert Opin Investig Drugs. 2012;21(6):879-889.

11. Grothey A, Van Cutsem E, Sobrero A, et al; CORRECT Study Group. Regorafenib monotherapy for previously treated metastatic colorectal cancer (CORRECT): an international, multicentre, randomised, placebocontrolled, phase 3 trial. Lancet. 2013;381(9863):303-312.

12. Demetri GD, Reichardt P, Kang YK, et al; GRID study investigators. Efficacy and safety of regorafenib for advanced gastrointestinal stromal tumours after failure of imatinib and sunitinib (GRID): an international, multicentre, randomised, placebo-controlled, phase 3 trial. Lancet. 2013;381(9863):295-302.

13. Bruix J, Tak WY, Gasbarrini A, et al. Regorafenib as second-line therapy for intermediate or advanced hepatocellular carcinoma: multicenter, open-label, phase II safety study. Eur J Cancer. 2013;49(16): 3412-3419.

14. Bruix J, Merle P, Granito A, et al. Efficacy and safety of regorafenib versus placebo in patients with hepatocellular carcinoma (HCC) progressing on sorafenib: results of the international, randomized phase 3 RESORCE trial. Ann Oncol. 2016;27(suppl 2):LBA-03.

15. Schlumberger M, Tahara M, Wirth LJ, et al. Lenvatinib versus placebo in radioiodine-refractory thyroid cancer. $N$ Engl J Med. 2015;372(7): 621-630.

16. Ikeda M, Okusaka T, Mitsunaga S, et al. Safety and pharmacokinetics of lenvatinib in patients with advanced hepatocellular carcinoma. Clin Cancer Res. 2016;22(6):1385-1394.

17. Fuchs CS, Tomasek J, Yong CJ, et al; REGARD Trial Investigators. Ramucirumab monotherapy for previously treated advanced gastric or gastro-oesophageal junction adenocarcinoma (REGARD): an international, randomised, multicentre, placebo-controlled, phase 3 trial. Lancet. 2014;383(9911):31-39.

18. Wilke H, Muro K, Van Cutsem E, et al; RAINBOW Study Group. Ramucirumab plus paclitaxel versus placebo plus paclitaxel in patients with previously treated advanced gastric or gastro-oesophageal junction adenocarcinoma (RAINBOW): a double-blind, randomised phase 3 trial. Lancet Oncol. 2014;15(11):1224-1235. 
19. Zhu AX, Park JO, Ryoo BY, et al; REACH Trial Investigators. Ramucirumab versus placebo as second-line treatment in patients with advanced hepatocellular carcinoma following first-line therapy with sorafenib (REACH): a randomised, double-blind, multicentre, phase 3 trial. Lancet Oncol. 2015;16(7):859-870.

20. Michalopoulos GK, De Frances MC. Liver regeneration. Science. 1997;276(5309):60-66.

21. Huelsken J, Vogel R, Erdmann B, et al. $\beta$-Catenin controls hair follicle morphogenesis and stem cell differentiation in the skin. Cell. 2001; 105(4):533-545.

22. Gherardi E, Birchmeier W, Birchmeier C, Vande Woude G. Targeting MET in cancer: rationale and progress. Nat Rev Cancer. 2012; 12(2):89-103.

23. Kondo S, Ojima H, Tsuda H, et al. Clinical impact of c-Met expression and its gene amplification in hepatocellular carcinoma. Int J Clin Oncol. 2013;18(2):207-213.

24. Eathiraj S, Palma R, Volckova E, et al. Discovery of a novel mode of protein kinase inhibition characterized by the mechanism of inhibition of human mesenchymal-epithelial transition factor (c-Met) protein autophosphorylation by ARQ 197. J Biol Chem. 2011;286(23):20666-20676.

25. Munshi N, Jeay S, Li Y, et al. ARQ 197, a novel and selective inhibitor of the human c-Met receptor tyrosine kinase with antitumor activity. Mol Cancer Ther. 2010;9(6):1544-1553.

26. Santoro A, Rimassa L, Borbath I, et al. Tivantinib for second-line treatment of advanced hepatocellular carcinoma: a randomised, placebocontrolled phase 2 study. Lancet Oncol. 2013;14(1):55-63.

27. Yakes FM, Chen J, Tan J, et al. Cabozantinib (XL184), a novel MET and VEGFR2 inhibitor, simultaneously suppresses metastasis, angiogenesis, and tumor growth. Mol Cancer Ther. 2011;10(12):2298-2308.

28. Elisei R, Schlumberger MJ, Müller SP, et al. Cabozantinib in progressive medullary thyroid cancer. J Clin Oncol. 2013;31(29):3639-3646.

29. Smith MR, De Bono JS, Cora N, et al. Final analysis of COMET-1: cabozantinib (Cabo) versus prednisone (Pred) in metastatic castrationresistant prostate cancer (mCRPC) patients (pts) previously treated with docetaxel (D) and abiraterone (A) and/or enzalutamide (E). J Clin Oncol. 2015;33(suppl 7):abstr139. [abstract].

30. Verslype C, Cohn AL, Kelley RK, et al. Activity of cabozantinib (XL184) in hepatocellular carcinoma: results from a phase II randomized discontinuation trial (RDT). J Clin Oncol. 2012;30:4007. [abstract].
31. Oquiñena S, Guillen-Grima F, Iñarrairaegui M, Zozaya JM, Sangro B. Spontaneous regression of hepatocellular carcinoma: a systematic review. Eur J Gastroenterol Hepatol. 2009;21(3):254-257.

32. Wang Z, Ke ZF, Lu XF, et al. The clue of a possible etiology about spontaneous regression of hepatocellular carcinoma: a perspective on pathology. Onco Targets Ther. 2015;8:395-400.

33. Palmer DH, Midgley RS, Mirza N, et al. A phase II study of adoptive immunotherapy using dendritic cells pulsed with tumor lysate in patients with hepatocellular carcinoma. Hepatology. 2009;49(1):124-132.

34. Hodi FS, O'Day SJ, McDermott DF, et al. Improved survival with ipilimumab in patients with metastatic melanoma. $N$ Engl J Med. 2010; 363(8):711-723.

35. Sangro B, Gomez-Martin C, de la Mata M, et al. A clinical trial of CTLA-4 blockade with tremelimumab in patients with hepatocellular carcinoma and chronic hepatitis C. J Hepatol. 2013;59(1):81-88.

36. Duffy AG, Makarova-Rusher OV, Pratt D, et al. Tremelimumab: a monoclonal antibody against CTLA-4 - in combination with subtotal ablation (trans catheter arterial chemoembolization (TACE), radiofrequency ablation (RFA) or cryoablation) in patients with hepatocellular carcinoma (HCC) and biliary tract carcinoma (BTC). J Clin Oncol. 2016;34(suppl):abstr4073.

37. Robert C, Long GV, Brady B, et al. Nivolumab in previously untreated melanoma without BRAF mutation. NEngl JMed. 2015;372(4):320-330.

38. Hamid O, Robert C, Daud A, et al. Safety and tumor responses with lambrolizumab (anti-PD-1) in melanoma. N Engl J Med. 2013;369(2): 134-144.

39. Melcher A, Parato K, Rooney CM, Bell JC. Thunder and lightning: immunotherapy and oncolytic viruses collide. Mol Ther. 2011;19(6): 1008-1016.

40. Jebar AH, Errington-Mais F, Vile RG, Selby PJ, Melcher AA, Griffin S. Progress in clinical oncolytic virus-based therapy for hepatocellular carcinoma. J Gen Virol. 2015;96(pt 7):1533-1550.

41. Heo J, Breitbach CJ, Moon A, et al. Sequential therapy with JX-594, a targeted oncolytic poxvirus, followed by sorafenib in hepatocellular carcinoma: preclinical and clinical demonstration of combination efficacy. Mol Ther. 2011;19(6):1170-1179.

42. Heo J, Reid T, Ruo L, et al. Randomized dose-finding clinical trial of oncolytic immunotherapeutic vaccinia JX-594 in liver cancer. Nat Med. 2013;19(3):329-336.
Journal of Hepatocellular Carcinoma

\section{Publish your work in this journal}

The Journal of Hepatocellular Carcinoma is an international, peerreviewed, open access journal that offers a platform for the dissemination and study of clinical, translational and basic research findings in this rapidly developing field. Development in areas including, but not limited to, epidemiology, vaccination, hepatitis therapy, pathology and

\section{Dovepress}

molecular tumor classification and prognostication are all considered for publication. The manuscript management system is completely online and includes a very quick and fair peer-review system, which is all easy to use. Visit http://www.dovepress.com/testimonials.php to read real quotes from published authors. 